\title{
Bioactive Components of Chinese Propolis Water Extract on Antitumor Activity and Quality Control
}

\author{
Hongzhuan Xuan, ${ }^{1,2}$ Yuehua Wang, ${ }^{2}$ Aifeng Li, ${ }^{3}$ Chongluo Fu, \\ Yuanjun Wang, ${ }^{2}$ and Wenjun Peng ${ }^{1}$ \\ ${ }^{1}$ Key Laboratory of Pollinating Insect Biology, Ministry of Agriculture, Beijing 100093, China \\ ${ }^{2}$ School of Life Science, Liaocheng University, Liaocheng 252059, China \\ ${ }^{3}$ College of Chemistry and Chemical Engineering, Liaocheng University, Liaocheng 252059, China \\ Correspondence should be addressed to Wenjun Peng; pengwenjun@vip.sina.com
}

Received 16 October 2015; Revised 25 January 2016; Accepted 7 March 2016

Academic Editor: Michael A. Savka

Copyright (C) 2016 Hongzhuan Xuan et al. This is an open access article distributed under the Creative Commons Attribution License, which permits unrestricted use, distribution, and reproduction in any medium, provided the original work is properly cited.

To understand the material basis of antitumor activity of Chinese propolis water extract (CPWE), we developed a simple and efficient method using macroporous absorptive resin coupled with preparative high performance liquid chromatography and separated and purified eleven chemical components (caffeic acid, ferulic acid, isoferulic acid, 3,4-dimethoxycinnamic acid, pinobanksin, caffeic acid benzyl ester, caffeic acid phenethyl ester, apigenin, pinocembrin, chrysin, and galangin) from CPWE; then we tested the antitumor activities of these eleven components using different human tumor cell lines (MCF-7, MDA-MB-231, HeLa, and A549). Furthermore, cell migration, procaspase 3 level, and reactive oxygen species (ROS) of effective components from CPWE were investigated. Our data showed that antitumor activities of the eleven components from CPWE were different from each other. CPWE and its effective components induced apoptosis by inhibiting tumor cell migration, activating caspase 3, and promoting ROS production. It can be deduced that the antitumor effects of propolis did not depend on a single component, and there must exist "bioactive components," which also provides a new idea for Chinese propolis quality control.

\section{Introduction}

Propolis is a resinous substance collected by Apis mellifera from various tree buds, and it has been used as a folk medicine since ancient time for its widely biological properties, such as antibacterial, antiviral, antioxidant, antiinflammatory, immunomodulatory, and antitumor. [1-5]. However, in propolis application the biggest problem is the instability of its therapeutic effects and the material basis has not been fully understood, which is caused by the imperfection of propolis quality control and evaluation system. Propolis quality control system is difficult to be established, for there are more than 600 constituents identified from different kinds of propolis in the world, such as polyphenols (flavonoids, phenolic acids, and their esters), terpenoids, steroids, and amino acids [6-8]. And the other major cause is that there is not a unified extract method and solvent process. Ethanol is the most common solvent during propolis extracting process, and most of the studies and biological activities in propolis are based on propolis ethanolic extract (PEE), which leads to little knowledge known about the biological activities of the propolis water extract, especially "poplar propolis" from China $[9,10]$.

Recently, we developed a simple and efficient method using macroporous absorptive resin (MAR) coupled with preparative high performance liquid chromatography (PHPLC) for separation of polyphenols from Chinese propolis water extract (CPWE). Six phenolic acids and five flavonoids (caffeic acid, ferulic acid, isoferulic acid, 3,4-dimethoxycinnamic acid, pinobanksin, caffeic acid benzyl ester, caffeic acid phenethyl ester (CAPE), apigenin, pinocembrin, chrysin, and galangin) with high purities were isolated, and the chemical structures were further confirmed by UV and NMR analysis [11]. 


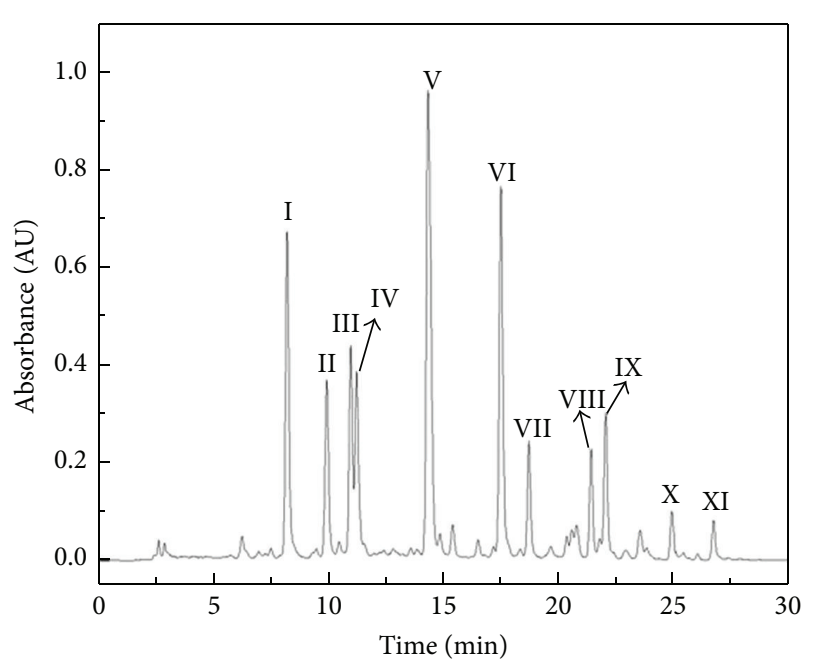

(a)

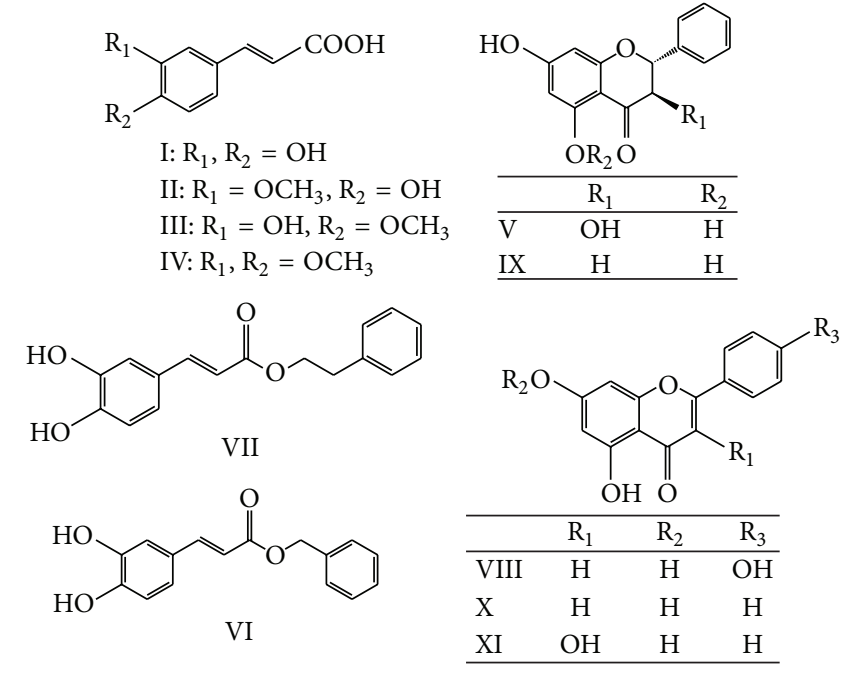

(b)

FIGURE 1: HPLC chromatograms of the crude Chinese propolis water extract (CPWE) and the chemical structure of the eleven components. I: caffeic acid, II: ferulic acid, III: isoferulic acid, IV: 3,4-dimethoxycinnamic acid, V: pinobanksin, VI: caffeic acid benzyl ester, VII: caffeic acid phenethyl ester, VIII: apigenin, IX: pinocembrin, X: chrysin, and XI: galangin.

Considering the imperfection of Chinese propolis quality control system and the ambiguity of material basis of antitumor activity of CPWE, in present study we studied the antitumor activities of CPWE and the eleven isolated components from CPWE to determine bioactive components of antitumor activity and provide a new idea for Chinese propolis quality control.

\section{Materials and Methods}

2.1. Chemicals and Reagents. Dulbecco's modified Eagle's medium (DMEM) was from Gibco (USA). Fetal bovine serum (FBS) was from Hyclone Lab Inc. (USA). Sulforhodamine B (SRB), Hoechst 33258, and 2', $7^{\prime}$-dichlorodihydrofluorescein (DCHF) were from Sigma Co. (USA). Acridine orange was from Amresco (USA). Primary antibodies against $\beta$-actin and secondary antibody (horseradish peroxidase) were from Santa Cruz Biotechnology (USA). Primary antibody against procaspase 3 was from Cell Signaling Technology (USA). All other reagents were ultrapure grade.

2.2. Preparation of Propolis Extracts. Chinese propolis was obtained from colonies of honeybees, A. mellifera L., in Shandong province of north China and the main plant origin was poplar (Populus sp.). Chinese propolis $0.25 \mathrm{~kg}$ was frozen, milled, and extracted with boiling water. The water extract was filtered, combined, and concentrated under reduced pressure with a rotary evaporator. Then $95 \%$ ethanol was added to the solution to remove polysaccharide until the concentration of ethanol was about $70 \%$. After $12 \mathrm{~h}$, the supernatant was separated and concentrated under reduced pressure. The water-soluble fraction was first "prefractioned" by MAR to obtain four subfractions; and they were all subjected to PHPLC to get different components [11].
2.3. Cell Culture. The human breast cancer cells, MCF-7 (human breast cancer ER (+)) and MDA-MB-231 (human breast cancer ER (-)) cells, lung cancer A549 cells, and human colonic carcinoma HeLa cells were purchased from American Type Culture Collection (ATCC, USA). MCF-7, MDA-MB-231, A549, and HeLa cells were cultured in DMEM medium supplemented with heat-inactivated $10 \%$ FBS and $100 \mathrm{U} / \mathrm{mL}$ of penicillin and $100 \mu \mathrm{g} / \mathrm{mL}$ streptomycin. Cells were incubated at $37^{\circ} \mathrm{C}$ in a humidified atmosphere of $5 \%$ $\mathrm{CO}_{2}$ and $95 \%$ air.

2.4. Cell Viability Assay. Four different tumor cells were seeded onto 96-well plates and treated with different components separated from CPWE $(20,40,80$, and $160 \mu \mathrm{M})$ for 24 and $48 \mathrm{~h}$, respectively. Cell viability was determined by SRB assay. In detail, fix cells by adding $100 \mu \mathrm{L}$ of cold $10 \%$ trichloroacetic acid and incubate for $1 \mathrm{~h}$ at $4^{\circ} \mathrm{C}$, and then wash the plates with deionized water five times. Add $50 \mu \mathrm{L}$ of $0.4 \%$ SRB solution to each well and shake for $5 \mathrm{~min}$ on titer plate shaker. Wash the plate with $1 \%$ acetate five times, and subsequently add $100 \mu \mathrm{L}$ of $10 \mathrm{mM}$ Tris base to dissolve the bound dye. Mix for 5 min on a microtiter plate shaker and read optical densities at the wavelength of $492 \mathrm{~nm}$ using Multiskan MK3 microplate reader (Thermo Co., USA). The viability (\%) was expressed as (OD of treated group/OD of control group) $\times 100 \%$. The viability of the control cells was set to $100 \%$.

2.5. Nuclear Fragmentation Assay. The morphological changes of nuclei of MCF-7 cells treated with different components from CPWE were detected by acridine orange staining. At $48 \mathrm{~h}$, cells were washed gently with $1 \mathrm{x}$ PBS once and then stained with $5 \mu \mathrm{g} / \mathrm{mL}$ acridine orange at room temperature for $1 \mathrm{~min}$, after that they were washed 


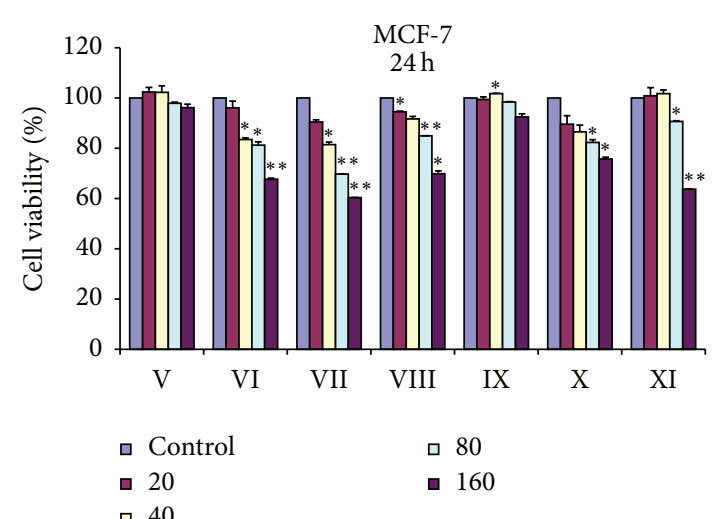

(a)

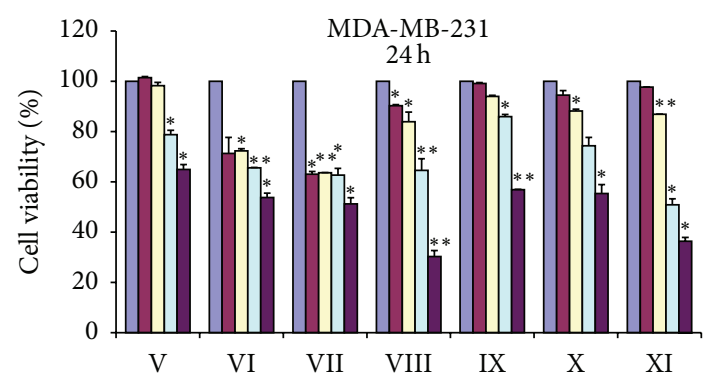

๑ Control

- 20

40

(c)

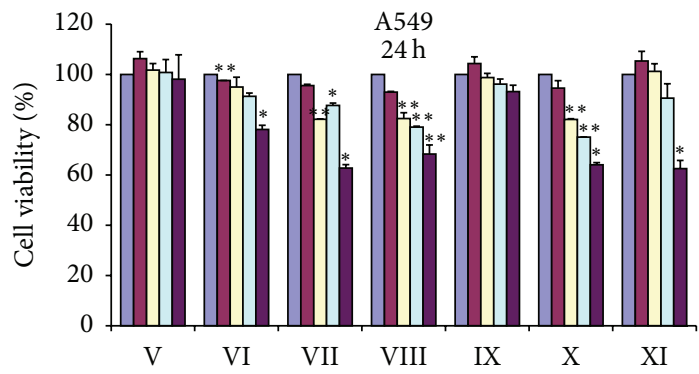

口 Control

- 20

40

口 80

- 160

(e)

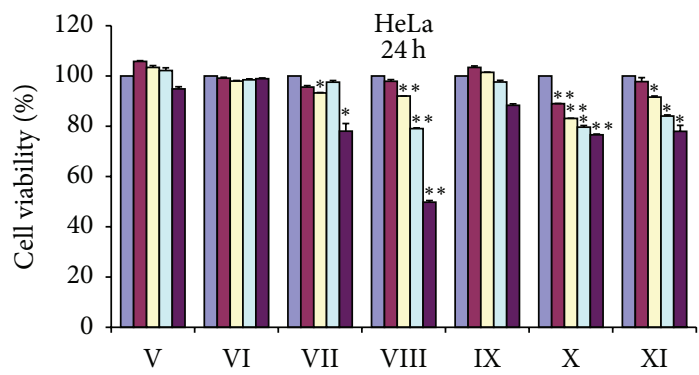

口 Control

- 20

40

80

- 160

(1)

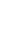




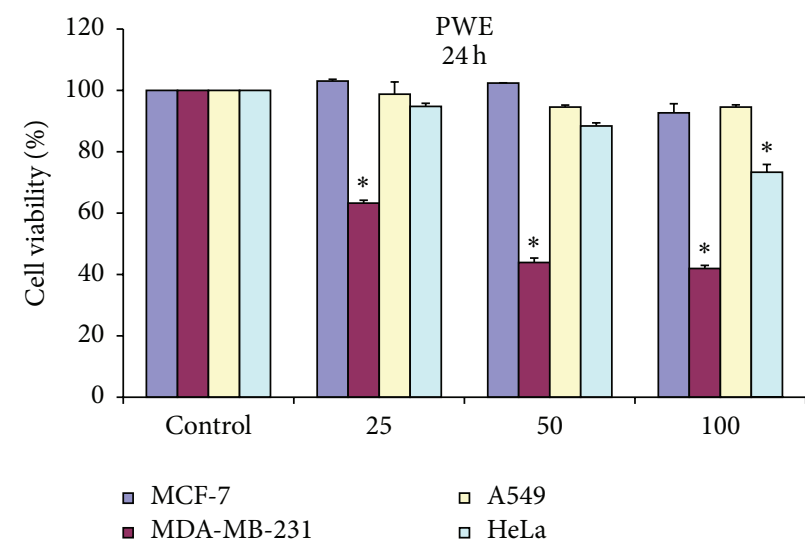

(i)

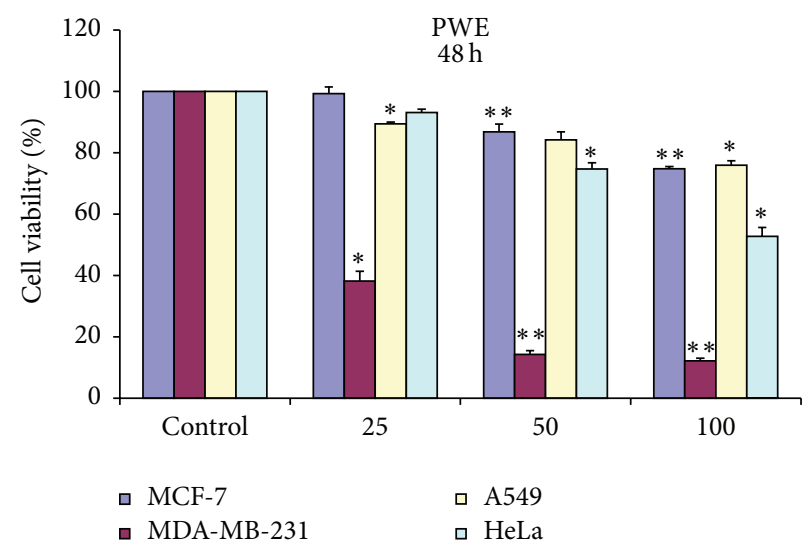

(j)

FIGURE 2: Effects of the seven components isolated from CPWE on the proliferation of four tumor cells. (a) and (b), effect of the seven components $(20,40,80$, and $160 \mu \mathrm{M})$ on MCF-7 cell viability at 24 and $48 \mathrm{~h}$. (c) and (d), effect of the seven components on MDA-MB-231 cell viability at 24 and $48 \mathrm{~h}$. (e) and (f), effect of the seven components on A549 cell viability at 24 and $48 \mathrm{~h}$. (g) and (h), effect of the seven components on viability of HeLa cell viability at 24 and $48 \mathrm{~h}$. (i) and (j), effect of crude CPWE on proliferation of four tumor cells. Cell viability was tested by SRB assay and illustrated in column figures $\left({ }^{*} P<0.05,{ }^{* *} P<0.01\right.$ versus control, $\left.n=3\right)$. Data are means \pm SEM.

gently twice to be observed under a TE2000S fluorescence microscope (Nikon, Japan).

2.6. Hoechst 33258 Staining. Hoechst 33258 staining was used to observe apoptotic morphology of MCF-7 cells treated with different components from CPWE. At $48 \mathrm{~h}$, cells in all groups were stained with $10 \mu \mathrm{g} / \mathrm{mL}$ Hoechst 33258 for $15 \mathrm{~min}$ and then were gently washed with $1 \mathrm{x}$ PBS once. Nuclear condensation and fragmentation were observed under a TE2000S fluorescence microscope (Nikon, Japan).

2.7. Wound-Healing Assay. MDA-MB-231 cells were grown to $80 \%$ confluence in a 24 -well plate; then the monolayers were scratched with a plastic tip, washed by $1 \mathrm{x}$ PBS to remove floating cell debris, and then incubated in medium in the absence or presence of different components from CPWE for $48 \mathrm{~h}$. Cell migration into the wound surface was determined under a TE2000S inverted microscope (Nikon, Japan). Migrated cells across the scratched lines were counted by Image-Pro Plus software (USA).

2.8. Western Blotting Analysis. Western blotting analysis was used to determine the protein levels in cells treated with different components from CPWE. Cells were collected and lysed in the lysis buffer, and protein concentration was measured by Bradford method as previously described [12]. Protein $(30 \mu \mathrm{g})$ was separated by running through $12 \%$ SDS-PAGE gel and transferred to the PVDF membrane. The transferred proteins were visualized with an enhanced chemiluminescence detection kit.

2.9. Measurement of ROS Production. ROS production in MCF-7 cells treated with different components from CPWE was determined by use of a fluorescent probe, DCHF as previously described [13]. The fluorescence was observed on a laser scanning confocal microscopy (Olympus FV1200, Japan). ROS level was quantified by Image-Pro Plus software (USA). Results were shown as relative fluorescence intensity of three independent experiments.

2.10. Statistical Analysis. All experiments were performed in duplicate and repeated at least 3 times. Data are expressed as means \pm SEM. Statistical analyses were performed using independent $t$-tests and analysis of variance (ANOVA) followed by the Tukey post hoc test. A $P<0.05$ was considered significant.

\section{Results}

3.1. Major Components of CPWE. Finally, eleven components from CPWE were obtained including I: caffeic acid (30 mg), II: ferulic acid (16 mg), III: isoferulic acid (10 mg), IV: 3,4-dimethoxycinnamic acid (12 mg), V: pinobanksin (42 mg), VI: caffeic acid benzyl ester $(36 \mathrm{mg})$, VII: caffeic acid phenethyl ester (12 mg), VIII: apigenin ( $8 \mathrm{mg})$, IX: pinocembrin (11 mg), X: chrysin (5 mg), and XI: galangin $(4 \mathrm{mg})$. Their purities were all above $98 \%$ as determined by HPLC, and the chemical structures (shown in Figure 1) were confirmed by UV and NMR analysis.

\subsection{Effects of the Eleven Components Isolated from CPWE} on the Proliferation of Four Tumor Cell Lines. We investigated the sensitivity of four tumor cell lines to the eleven components $(20,40,80$, and $160 \mu \mathrm{M})$ and $\operatorname{CPWE}(25,50$, and $100 \mu \mathrm{g} / \mathrm{mL}$ ) for 24 and $48 \mathrm{~h}$ using SRB assay at 24 and $48 \mathrm{~h}$. Caffeic acid, ferulic acid, isoferulic acid, and 3,4dimethoxycinnamic acid had no significant cytotoxicity to four tumor cells (data were not shown); the other seven components significantly inhibited four tumor cells' proliferation in a dose- and time-dependent manner. The crude CPWE also inhibited cell proliferation of four tumor cells; however, 

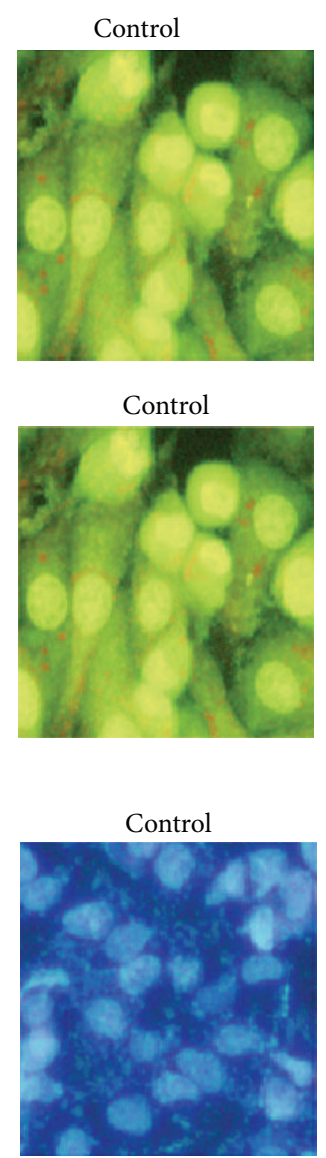

Control

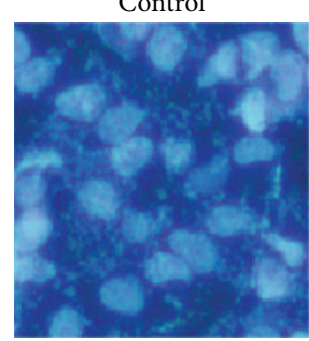

V

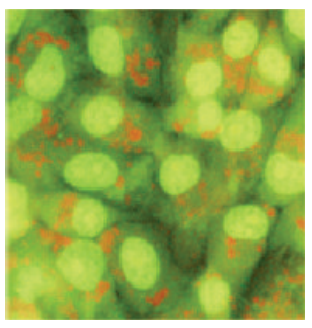

IX

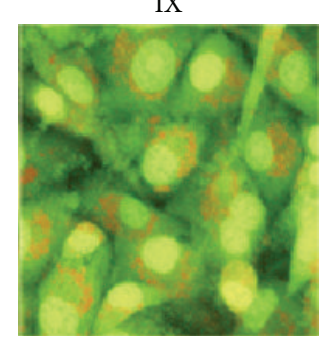

V

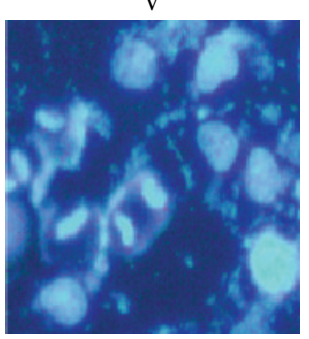

IX

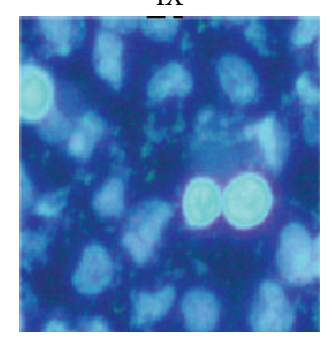

VI

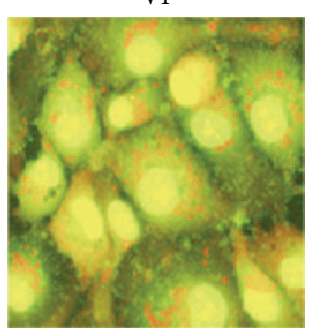

X

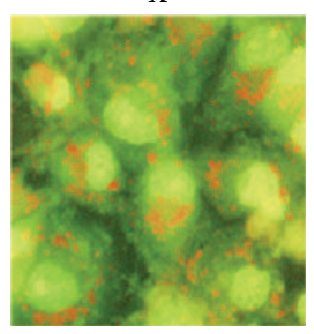

(a)

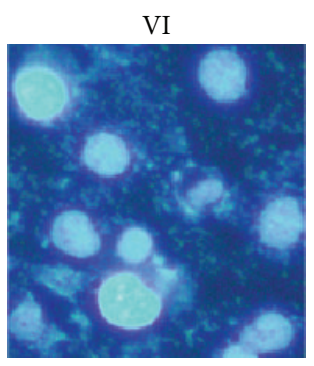

$\mathrm{X}$

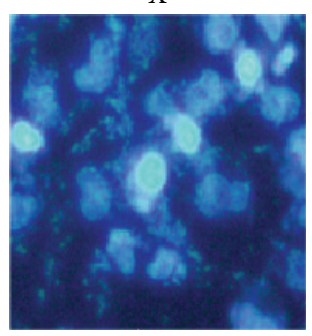

(b)
VII

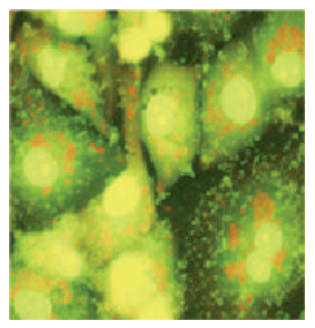

XI
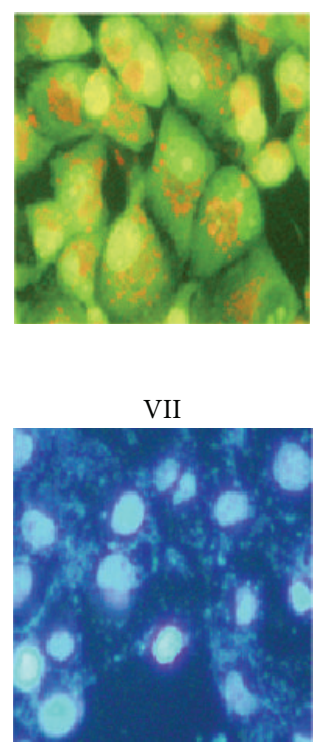

XI

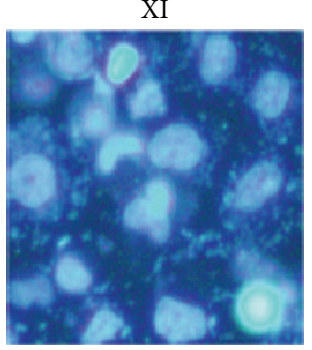

VIII

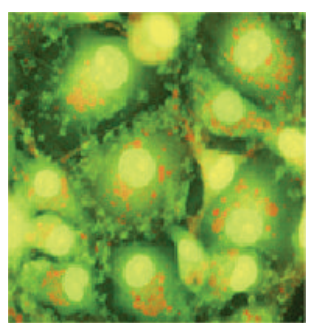

PWE
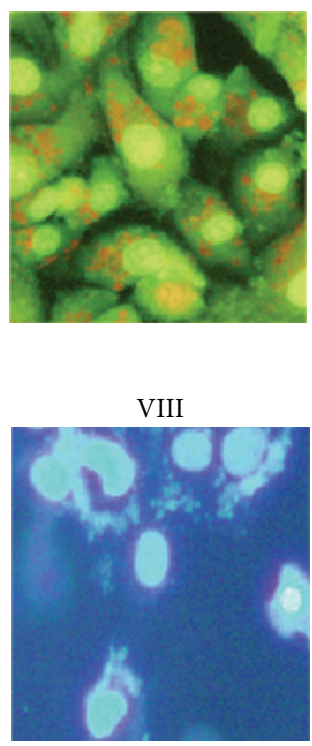

PWE

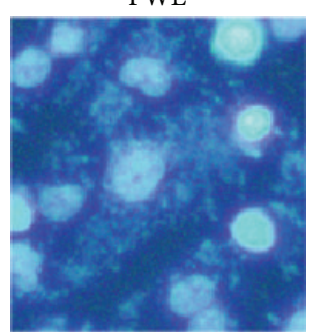

Figure 3: Effects of the seven components and crude CPWE on nuclear fragmentations of MCF-7 cells. (a) Morphological changes of nuclei by staining with acridine orange at $48 \mathrm{~h}(\times 200)$. (b) Morphological changes of nuclei by staining with Hoechst 33258 at $48 \mathrm{~h}(\times 200)$.

the inhibitory effect of CPWE was lower than that of ethanolextracted Chinese propolis, which was tested previously [14]. Furthermore, the sensitivity of four tumor cell lines to the seven components and CPWE from strong to weak was followed by MDA-MB-231, HeLa, A549, and MCF-7 cells $\left({ }^{*} P<0.05,{ }^{* *} P<0.01\right.$; Figure 2$)$.

Notably, the cytotoxicity of pinocembrin to tumor cells was higher than pinobanksin; CAPE was higher than caffeic acid benzyl ester, and the most effective antitumor concentration for seven different components was at concentration higher than $80 \mu \mathrm{M}$, so we used $80 \mu \mathrm{M}$ for the seven components and CPWE $100 \mu \mathrm{g} / \mathrm{mL}$ as the following study dose.
3.3. Effects of the Seven Different Components and CPWE on Apoptosis in MCF-7 Cells. Acridine orange staining and Hoechst 33258 staining results indicated that the seven different components at concentration of $80 \mu \mathrm{M}$ and CPWE $(100 \mu \mathrm{g} / \mathrm{mL})$ evidently induced nuclear condensation and fragmentation in MCF-7 cells (Figure 3).

3.4. Effects of the Seven Different Components and CPWE on MDA-MB-231 Cells Migration. The migrations of MDA-MB231 cells were detected by wound-healing assay after being treated with the seven different components at concentration 

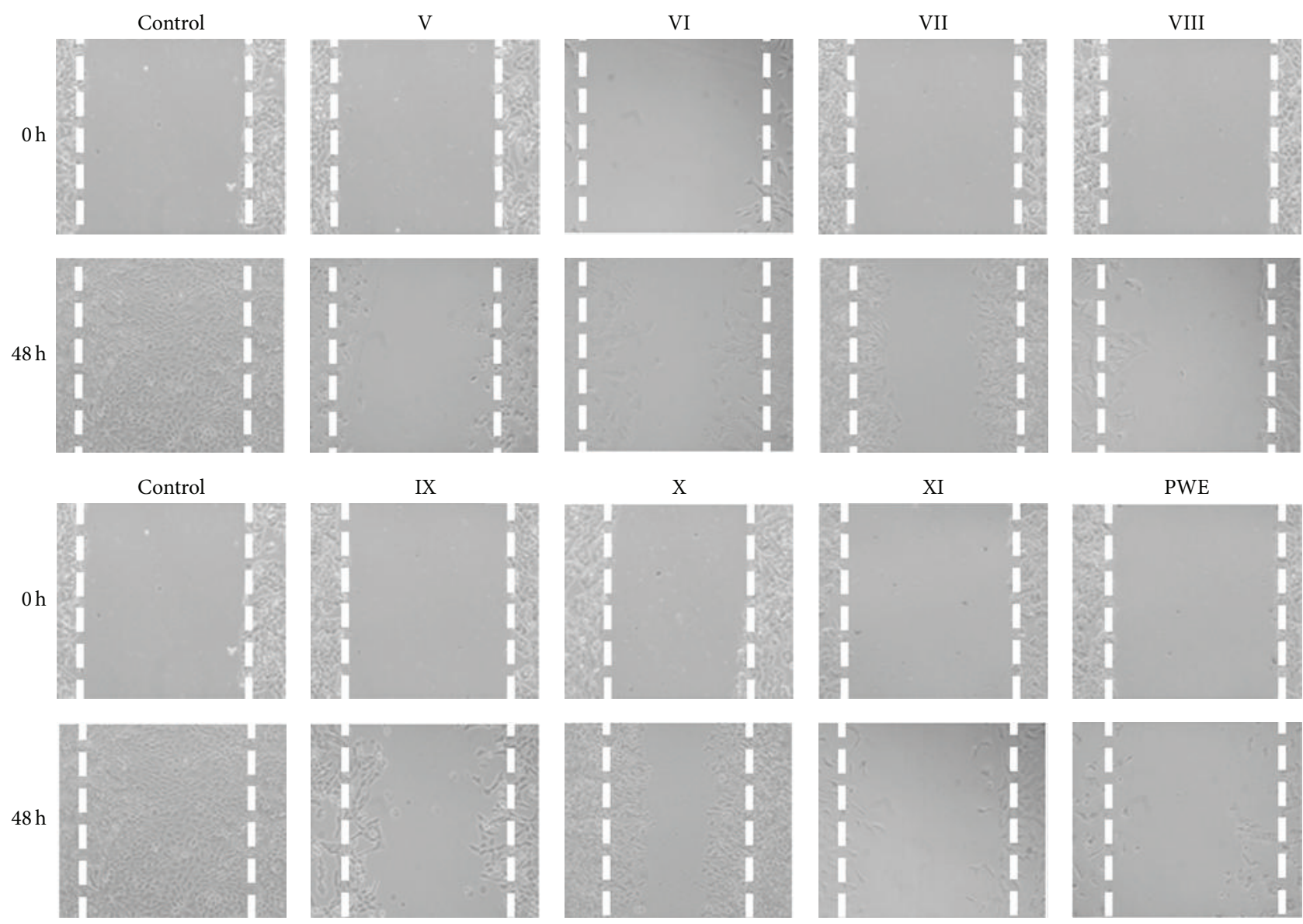

(a)

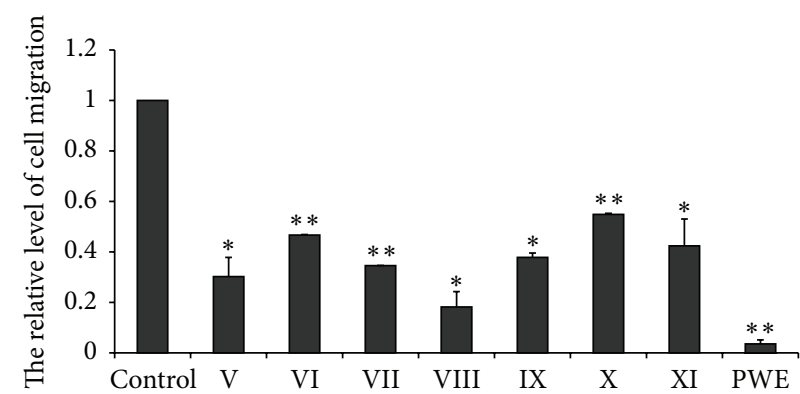

(b)

Figure 4: Effects of the seven components and crude CPWE on migration of MDA-MB-231 cells. (a) Cell migration micrographs obtained under a phase contrast microscope at 0 and $48 \mathrm{~h}(\times 100)$. (b) Relative levels of cell migration $\left({ }^{*} P<0.05,{ }^{* *} P<0.01\right.$ versus control, $\left.n=3\right)$.

of $80 \mu \mathrm{M}$ and CPWE $(100 \mu \mathrm{g} / \mathrm{mL})$; the results indicated that the seven different components and CPWE significantly inhibited MDA-MB-231 cells migration at $48 \mathrm{~h}$ (Figure 4).

3.5. Effects of the Seven Different Components and CPWE on the Level of Procaspase 3 in Two Breast Cancer Cells. The seven different components at concentration of $80 \mu \mathrm{M}$ and CPWE $(100 \mu \mathrm{g} / \mathrm{mL})$ significantly activated caspase 3 by western blotting assay in MCF-7 and MDA-MB-231 cells (Figure 5).
3.6. Effects of the Seven Different Components and CPWE on the Production of ROS in MCF-7 Cells. The seven different components at concentration of $80 \mu \mathrm{M}$ and CPWE $(100 \mu \mathrm{g} / \mathrm{mL})$ obviously affected ROS production in MCF-7 cells although the ROS levels were different from each other (Figure 6).

\section{Discussion}

Previous studies from our group reported the biological activities of Chinese propolis [15-17] and the present study 


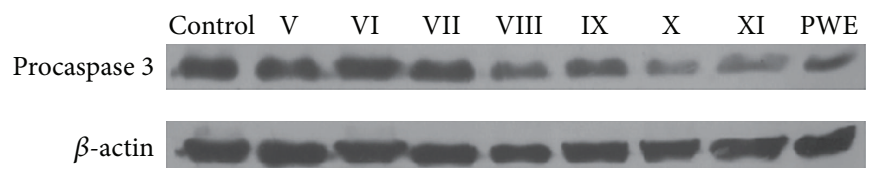

(a)

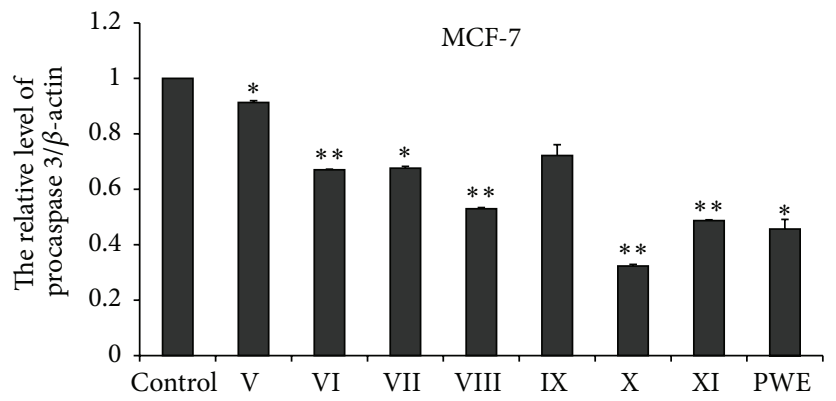

- Procaspase $3 / \beta$-actin

(b)

$\begin{array}{llllllllllll}\text { Control } & \text { V } & \text { VI } & \text { VII } & \text { VIII } & \text { IX } & \text { X } & \text { XI } & \text { PWE }\end{array}$

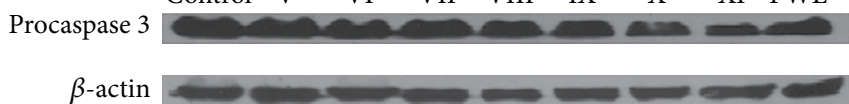

(c)

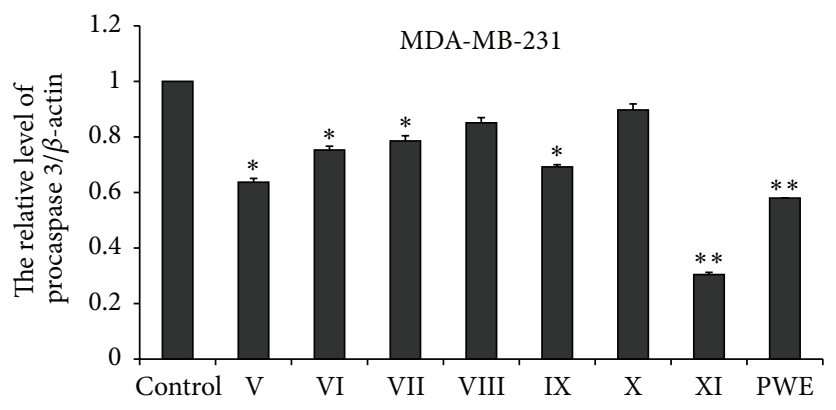

- Procaspase $3 / \beta$-actin

(d)

Figure 5: Effects of the seven components and crude CPWE on the expression of procaspase 3 in MCF-7 and MDA-MB-231 cells. (a) and (c), expression of procaspase 3 in MCF-7 and MDA-MB-231 cells at $24 \mathrm{~h}$, respectively; (b) and (d) quantification of relative expression quantity in MCF-7 and MDA-MB-231 cells at $24 \mathrm{~h}$, respectively $\left({ }^{*} P<0.05,{ }^{* *} P<0.01\right.$ versus control, $\left.n=3\right)$.

was the first one to investigate the effective components on antitumor activity in CPWE. Four phenolic acids (caffeic acid, ferulic acid, isoferulic acid, and 3,4-dimethoxycinnamic acid) had little cytotoxicity on four tumor cell lines; the other seven constituents (pinobanksin, caffeic acid benzyl ester, caffeic acid phenethyl ester, apigenin, pinocembrin, chrysin, and galangin) obviously decreased four tumor cells' proliferation, although the inhibitory effects of the seven components were different from each other, which indicated that the antitumor effects of CPWE did not depend on a single component, and at least the seven effective components might be "bioactive components" of antitumor activity. Admittedly, there must be other effective components needed to be studied further.
The standardization of Chinese propolis has caused some interest in recent years in China, and HPLC fingerprint of Chinese propolis from different regions, sources, and seasons has been fully studied, and the authentication standard of Chinese propolis and poplar buds had also been established $[18,19]$, which greatly promoted the research of quality control system of Chinese propolis. It was pointed out that chrysin, catechol, or another component from propolis could be a candidate for the standardization of Chinese propolis $[20,21]$. However, there still exist a lot of problems. For example, propolis has similar biological activities although chemical components vary greatly [22]. And more importantly, a number of studies have confirmed that propolis and its plant sources, poplar buds or gums, have similar biological 

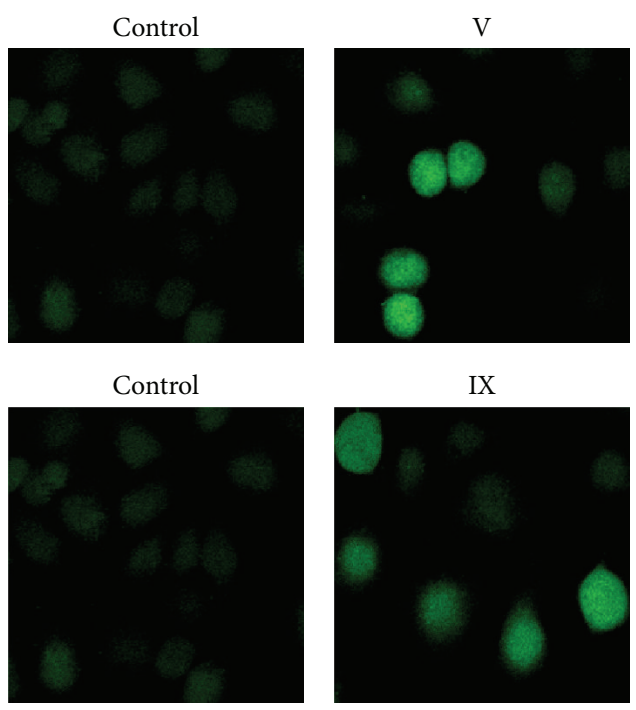

IX
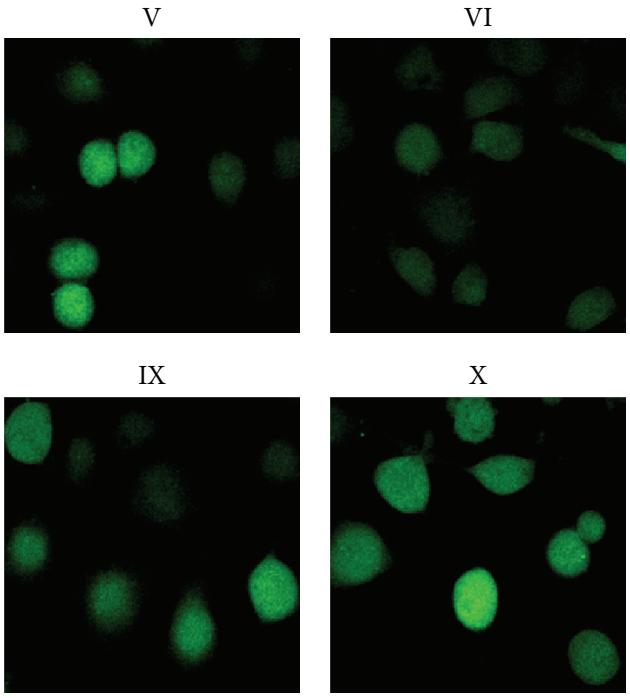

$\mathrm{X}$

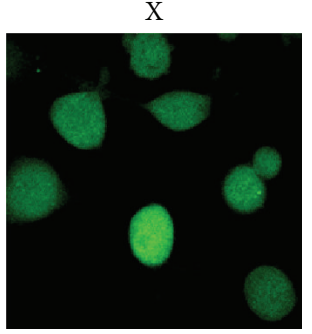

(a)

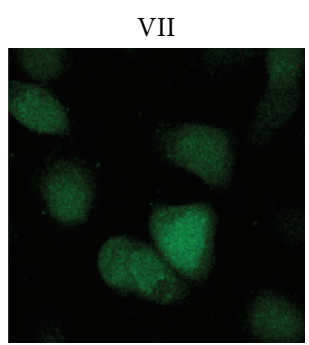

XI
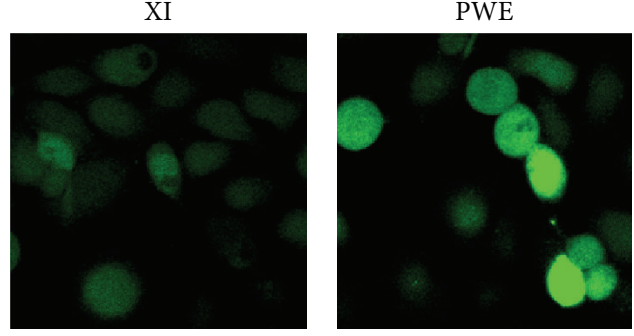

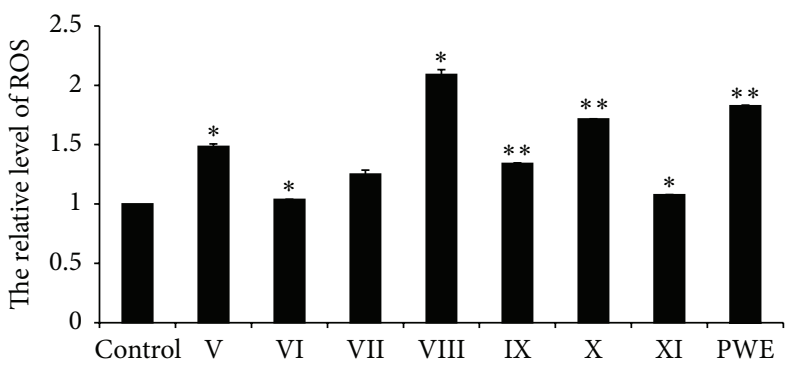

(b)

FIGURE 6: Effects of the seven components and crude CPWE on the production of reactive oxygen species (ROS) in MCF-7 cells. (a) Fluorescent micrographs obtained at $48 \mathrm{~h}$. (b) Quantification of relative quantity of ROS in MCF-7 cells. Values represent the relative fluorescent intensity per cell determined by laser scanning confocal microscopy $\left({ }^{*} P<0.05,{ }^{* *} P<0.01\right.$ versus control, $\left.n=3\right)$.

activities. Wang et al. indicated that ethanol extracts of Chinese propolis (EECP) and buds from poplar had similar anti-inflammatory effects in vivo and in vitro [23]. Another report suggested that the antioxidant mechanisms of EECP and poplar gums were similar, but they also indicated that the antioxidant activities of EECP were stronger than poplar gums. Further analysis indicated that the total content of eight components from EECP (caffeic acid, ferulic acid, $p$ coumaric acid, apigenin, chrysin, pinocembrin, CAPE, and galangin) was $5.85 \mathrm{~g} / 100 \mathrm{~g}$. However, in poplar gums, caffeic acid, ferulic acid, and $p$-coumaric acid were not identified, and the total content of the other five components was only $2.59 \mathrm{~g} / 100 \mathrm{~g}$ [24]. Based on the facts we deduced that the major cause for EECP with a higher antioxidant than poplar gums was that EECP had more effective components. In present study, we further confirmed that it was not a single component playing the antitumor activity in propolis. Thus, the quality evaluation system of Chinese propolis might be imperfect if it is only based on the quantitative analysis of chemical composition of propolis or some single component, and here we proposed that it was acceptable to perfect the quality evaluation system of Chinese propolis based on "bioactive components."

The major mechanism of inhibiting tumor cell proliferation of the seven effective components from CPWE was to induce apoptosis by activating caspase 3 , the executor of apoptosis, and induce ROS production, which was consistent with our previous studies [14].

In summary, our data highlight the effective components of CPWE on antitumor activity and the probable action mechanisms in inhibiting tumor cell proliferation and provide a novel idea for Chinese propolis quality control.

\section{Competing Interests}

The authors declare that they have no competing interests.

\section{Acknowledgments}

This work was supported by the grants from the National Natural Science Foundation of China (no. 31201860); Shandong Provincial Natural Science Foundation of China (no. 
ZR2012CQ003); Open foundation of Key Laboratory of Pollinating Insect Biology, Ministry of Agriculture, China (no. 2015MFNZS02); and the College Student Innovation Foundation of Liaocheng, University of China (nos. SF2014268, 26312150906).

\section{References}

[1] G. C.-F. Chan, K.-W. Cheung, and D. M.-Y. Sze, "The immunomodulatory and anticancer properties of propolis," Clinical Reviews in Allergy and Immunology, vol. 44, no. 3, pp. 262-273, 2013.

[2] S. Patel, "Emerging adjuvant therapy for cancer: propolis and its constituents," Journal of Dietary Supplements, vol. 13, no. 3, pp. 245-268, 2016.

[3] J. M. Sforcin and V. Bankova, "Propolis: is there a potential for the development of new drugs?" Journal of Ethnopharmacology, vol. 133, no. 2, pp. 253-260, 2011.

[4] G. Valenzuela-Barra, C. Castro, C. Figueroa et al., "Antiinflammatory activity and phenolic profile of propolis from two locations in Región Metropolitana de Santiago, Chile," Journal of Ethnopharmacology, vol. 168, pp. 37-44, 2015.

[5] S. Ishiai, W. Tahara, E. Yamamoto, R. Yamamoto, and K. Nagai, "Histone deacetylase inhibitory effect of Brazilian propolis and its association with the antitumor effect in Neuro2a cells," Food Science \& Nutrition, vol. 2, no. 5, pp. 565-570, 2014.

[6] M. Duman and E. Özpolat, "Effects of water extract of propolis on fresh shibuta (Barbus grypus) fillets during chilled storage," Food Chemistry, vol. 189, Article ID 16314, pp. 80-85, 2015.

[7] V. Bankova, "Chemical diversity of propolis and the problem of standardization," Journal of Ethnopharmacology, vol. 100, no. 12, pp. 114-117, 2005.

[8] S. Huang, C.-P. Zhang, K. Wang, G. Q. Li, and F.-L. Hu, "Recent advances in the chemical composition of propolis," Molecules, vol. 19, no. 12, pp. 19610-19632, 2014.

[9] F. Hu, H. R. Hepburn, Y. Li, M. Chen, S. E. Radloff, and S. Daya, "Effects of ethanol and water extracts of propolis (bee glue) on acute inflammatory animal models," Journal of Ethnopharmacology, vol. 100, no. 3, pp. 276-283, 2005.

[10] T. D. Kwon, M. W. Lee, and K. H. Kim, "The effect of exercise training and water extract from propolis intake on the antioxidant enzymes activity of skeletal muscle and liver in rat," Journal of Exercise Nutrition and Biochemistry, vol. 18, no. 1, pp. 9-17, 2014.

[11] A. Li, H. Xuan, A. Sun, R. Liu, and J. Cui, "Preparative separation of polyphenols from water-soluble fraction of Chinese propolis using macroporous absorptive resin coupled with preparative high performance liquid chromatography," Journal of Chromatography B, vol. 1012-1013, pp. 42-49, 2016.

[12] A.-Y. Du, B.-X. Zhao, D.-L. Yin, S.-L. Zhang, and J.-Y. Miao, "Discovery of a novel small molecule, 1-ethoxy-3-(3,4methylenedioxyphenyl)-2-propanol, that induces apoptosis in A549 human lung cancer cells," Bioorganic and Medicinal Chemistry, vol. 13, no. 13, pp. 4176-4183, 2005.

[13] N. Suematsu, H. Tsutsui, J. Wen et al., "Oxidative stress mediates tumor necrosis factor- $\alpha$-induced mitochondrial DNA damage and dysfunction in cardiac myocytes," Circulation, vol. 107, no. 10, pp. 1418-1423, 2003.

[14] H. Xuan, Z. Li, H. Yan et al., "Antitumor activity of Chinese propolis in human breast cancer MCF-7 and MDAMB-231 cells," Evidence-Based Complementary and Alternative Medicine, vol. 2014, Article ID 280120, 11 pages, 2014.
[15] Y.-J. Li, H.-Z. Xuan, Q.-Y. Shou, Z.-G. Zhan, X. Lu, and F.-L. $\mathrm{Hu}$, "Therapeutic effects of propolis essential oil on anxiety of restraint-stressed mice," Human and Experimental Toxicology, vol. 31, no. 2, pp. 157-165, 2012.

[16] H. Xuan, J. Zhao, J. Miao, Y. Li, Y. Chu, and F. Hu, "Effect of Brazilian propolis on human umbilical vein endothelial cell apoptosis," Food and Chemical Toxicology, vol. 49, no. 1, pp. 7885, 2011.

[17] H. U. Fuliang, H. R. Hepburn, H. Xuan, M. Chen, S. Daya, and S. E. Radloff, "Effects of propolis on blood glucose, blood lipid and free radicals in rats with diabetes mellitus," Pharmacological Research, vol. 51, no. 2, pp. 147-152, 2005.

[18] J. Zhou, Y. Li, J. Zhao, X. Xue, L. Wu, and F. Chen, "Geographical traceability of propolis by high-performance liquidchromatography fingerprints," Food Chemistry, vol. 108, no. 2, pp. 749-759, 2008.

[19] Z. Cui-Ping, H. Shuai, W. Wen-Ting et al., "Development of high-performance liquid chromatographic for quality and authenticity control of Chinese propolis," Journal of Food Science, vol. 79, no. 7, pp. C1315-C1322, 2014.

[20] S. Huang, C.-P. Zhang, G. Q. Li, Y.-Y. Sun, K. Wang, and F.-L. $\mathrm{Hu}$, "Identification of catechol as a new marker for detecting propolis adulteration," Molecules, vol. 19, no. 7, pp. 10208-10217, 2014.

[21] L.-P. Sun, A.-L. Chen, H.-C. Hung et al., "Chrysin: a histone deacetylase 8 inhibitor with anticancer activity and a suitable candidate for the standardization of Chinese propolis," Journal of Agricultural and Food Chemistry, vol. 60, no. 47, pp. 1174811758, 2012.

[22] W. Zhu, Y.-H. Li, M.-L. Chen, and F.-L. Hu, "Protective effects of Chinese and Brazilian propolis treatment against hepatorenal lesion in diabetic rats," Human and Experimental Toxicology, vol. 30, no. 9, pp. 1246-1255, 2011.

[23] K. Wang, J. Zhang, S. Ping et al., "Anti-inflammatory effects of ethanol extracts of Chinese propolis and buds from poplar (Populus $\times$ canadensis)," Journal of Ethnopharmacology, vol. 155, no. 1, pp. 300-311, 2014.

[24] J. Zhang, X. Cao, S. Ping et al., "Comparisons of ethanol extracts of Chinese propolis (poplar type) and poplar gums based on the antioxidant activities and molecular mechanism," Evidence-Based Complementary and Alternative Medicine, vol. 2015, Article ID 307594, 15 pages, 2015. 


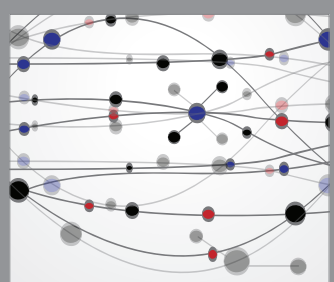

The Scientific World Journal
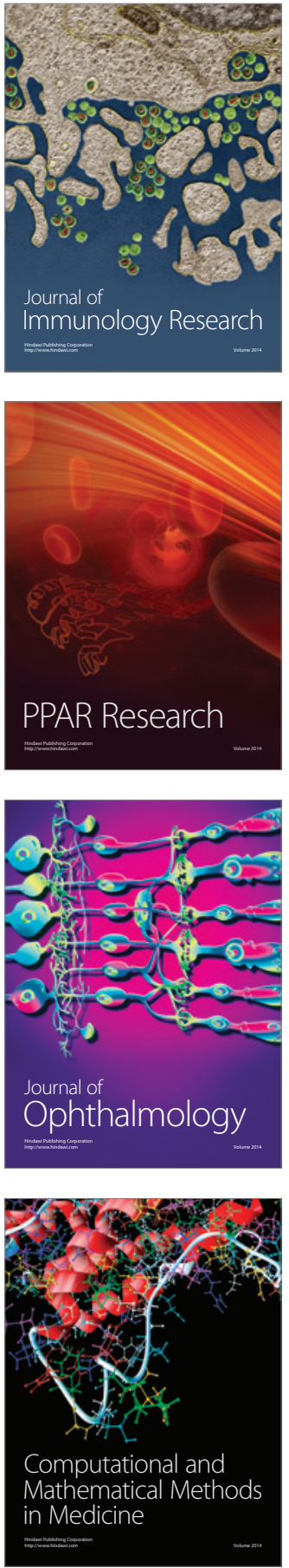

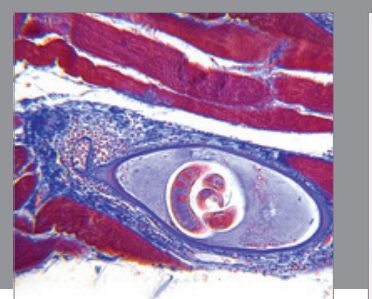

Gastroenterology Research and Practice

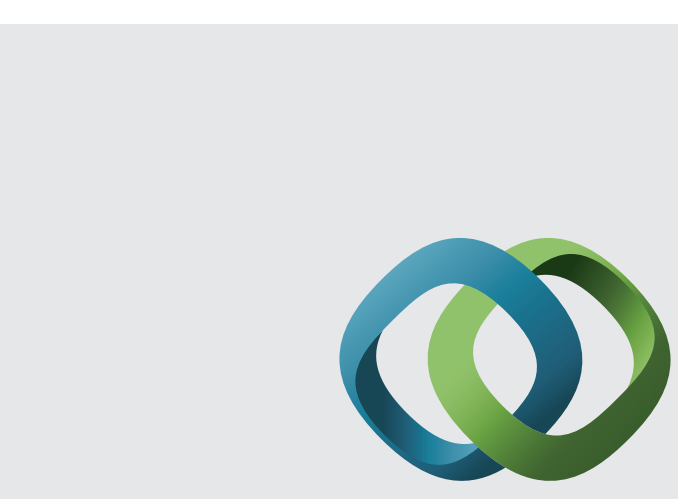

\section{Hindawi}

Submit your manuscripts at

http://www.hindawi.com
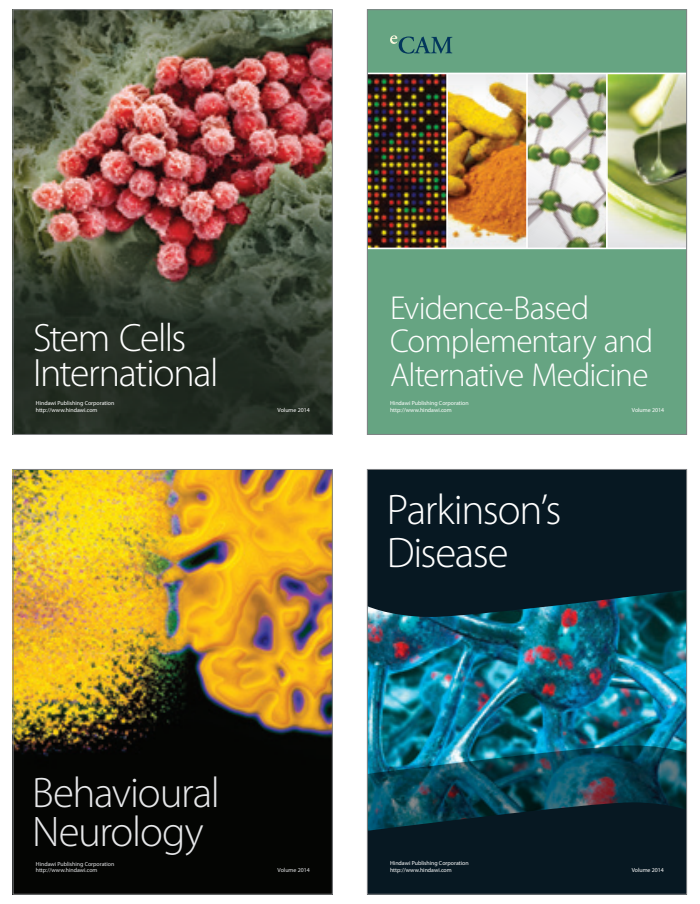
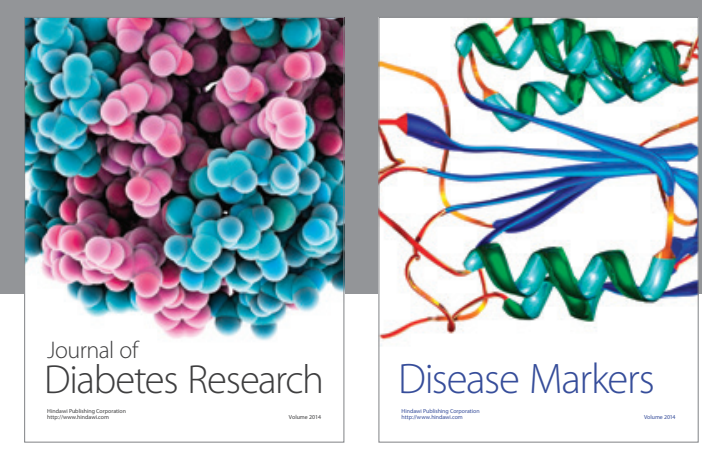

Disease Markers
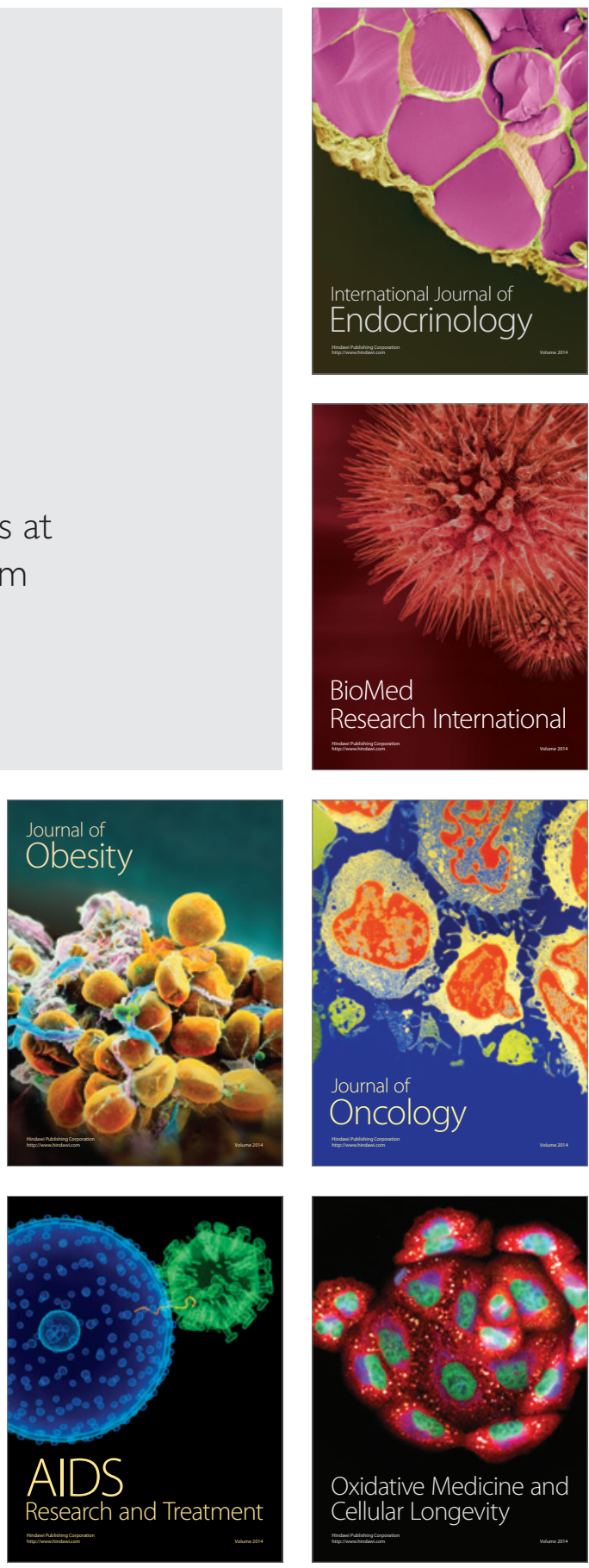